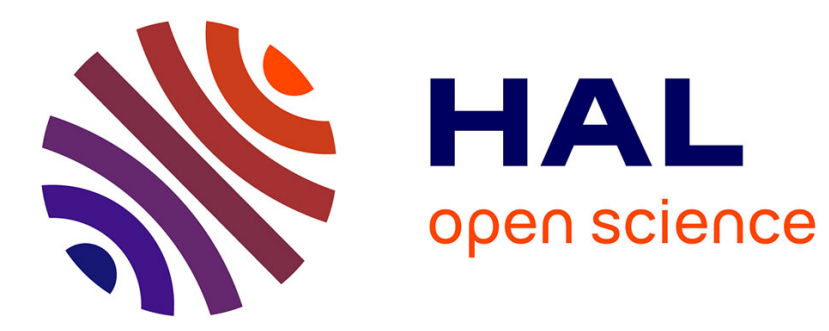

\title{
Compensation des effets mécaniques dans un cristal de coupe SC
}

\author{
R. Delaite, J.-P. Valentin
}

\section{To cite this version:}

R. Delaite, J.-P. Valentin. Compensation des effets mécaniques dans un cristal de coupe SC. Revue de

Physique Appliquée, 1987, 22 (2), pp.119-123. 10.1051/rphysap:01987002202011900 . jpa-00245523

\section{HAL Id: jpa-00245523 \\ https://hal.science/jpa-00245523}

Submitted on 1 Jan 1987

HAL is a multi-disciplinary open access archive for the deposit and dissemination of scientific research documents, whether they are published or not. The documents may come from teaching and research institutions in France or abroad, or from public or private research centers.
L'archive ouverte pluridisciplinaire HAL, est destinée au dépôt et à la diffusion de documents scientifiques de niveau recherche, publiés ou non, émanant des établissements d'enseignement et de recherche français ou étrangers, des laboratoires publics ou privés. 


\title{
Compensation des effets mécaniques dans un cristal de coupe SC
}

\author{
R. Delaite et J.-P. Valentin
}

Laboratoire de Chronométrie, Electronique et Piézoélectricité, Ecole Nationale Supérieure de Mécanique et des Microtechniques, La Bouloie, route de Gray, 25030 Besançon Cedex, France

(Reçu le 11 juillet 1986, révisé le 10 octobre, accepté le 7 novembre 1986)

\begin{abstract}
Résumé. - Les effets d'une compression et d'une flexion sont étudiés dans le cas d'un cristal de quartz de coupe SC. L'existence de zones d'insensibilité est mise en évidence. Une nouvelle structure de maintien d'un résonateur de coupe SC est proposée.
\end{abstract}

Abstract. - In the case of SC cut quartz crystal the effects of compression and flexure stress are studied. Some stress-unsensitive areas are pointed out, and a new holdering structure is proposed.

\section{Introduction.}

Pour quelques applications spécifiques, il est possible de réaliser des suspensions fluides, magnétiques ou électrostatiques qui éliminent tout contact « solidesolide " entre le support et l'objet suspendu. Dans le cas d'un cristal résonnant monté en boîtier sous vide, de telles suspensions ne sont pas possibles. Il est nécessaire de passer par l'intermédiaire d'une fixation mécanique. Dès lors, des contraintes mécaniques évolutives, d'origines diverses, sont appliquées au cristal, engendrant une variation de la fréquence du résonateur.

Quatre phénomènes indépendants sont à l'origine de l'évolution des contraintes appliquées au cristal:

a) La relaxation des pré-contraintes du cristal et de son support, induites par la structure de maintien au cours du montage du résonateur.

b) Les déformations du cristal et de son support, liées à une variation de la température du résonateur (l'anisotropie cristalline complique la maîtrise de ce phénomène).

c) Les déformations du boîtier sous l'effet d'une variation de la pression extérieure appliquée au résonateur.

d) Les variations d'intensité ou d'orientation du champ accélérométrique auquel est soumis le résonateur.

Ce dernier phénomène est à l'origine de la sensibilité accélérométrique des résonateurs à quartz, sensibilité qui se manifeste aussi bien lors de l'application d'une accélération continue (résonateurs embarqués), variable (vibrations aléatoires et sinusoïdales) ou quasi- instantanée (chocs), que lors d'un simple changement de position du résonateur dans le champ accélérométrique auquel il est soumis (champ de pesanteur terrestre par exemple) [1].

Deux types de solutions peuvent être envisagés pour diminuer, voire annuler, l'influence des contraintes mécaniques évolutives sur la fréquence des résonateurs à cristal : la suspension par fils ou l'utilisation, pour une coupe cristalline donnée, de zones du cristal insensibles aux contraintes mécaniques. La suspension par fils a déjà été largement utilisée : sa mise en ouvre est délicate, le montage reste fragile et sensible aux vibrations, en particulier jusqu'à $1 \mathrm{kHz}$. L'utilisation de points de fixation insensibles aux contraintes $[2,3]$ ainsi que l'autosuspension [4] ont été proposées, mais récemment, il a été montré qu'une suspension par secteurs angulaires périphériques permettait, dans le cas de la coupe AT, de s'affranchir presque totalement des effets d'une variation des contraintes mécaniques appliquées au cristal résonnant [5].

Le but de cet article est l'étude des zones insensibles aux contraintes dans le cas de la coupe à double rotation dite coupe SC, coupe dont les nombreux avantages tendent à en généraliser l'emploi [6].

\section{Sensibilité à la compression.}

2.1 COEFFICIENT DE SENSIBILITÉ. - Un montage classique du cristal résonnant consiste à maintenir le cristal à l'aide de deux lames-supports soudées à l'embase du boîtier et collées de façon diamétralement opposée, sur la tranche de la lame cristalline. Tout naturellement, l'étude de la sensibilité des coupes 
cristallines à un couple de forces égales ou opposées suivant un diamètre a été faite pour les coupes les plus utilisées (Fig. 1). J. M. Ratajski [7] a, en particulier,

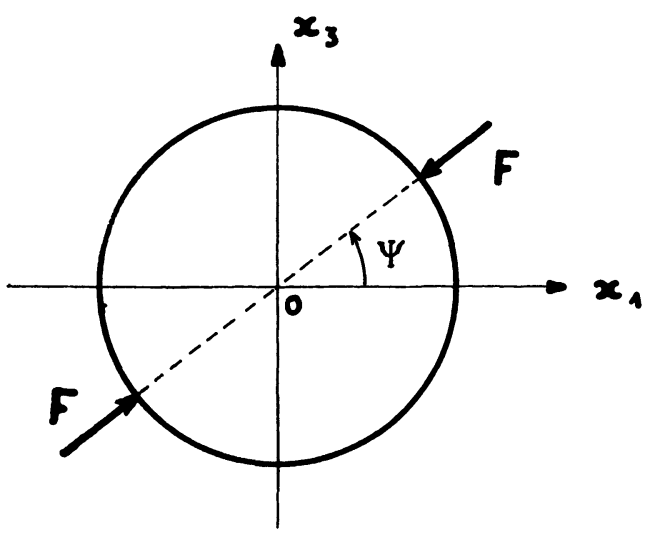

Fig. 1. - Cristal soumis à un couple de forces de compression.

[Strengths to a cristai.]

introduit un coefficient de sensibilité à un tel couple de forces, défini par la relation :

$$
K_{f}(\theta, \varphi, \Psi)=\frac{1}{F} \frac{\Delta f}{f} \frac{n D}{f}
$$

où $\theta$ et $\varphi$ sont les angles de coupe cristallographique de la lame considérée, $\Psi$ l'angle d'application du couple de forces $F, \Delta f$ la variation de fréquence induite par l'application du couple de forces, $f$ la fréquence de résonance, $n$ le rang de partiel de la vibration étudiée et $D$ le diamètre du cristal résonnant. Lorsque l'application du couple de forces $F$ n'est plus ponctuelle mais est effectuée suivant un secteur angulaire d'ouverture $2 \alpha$, la formulation précédente n'est plus suffisante (Fig. 2).

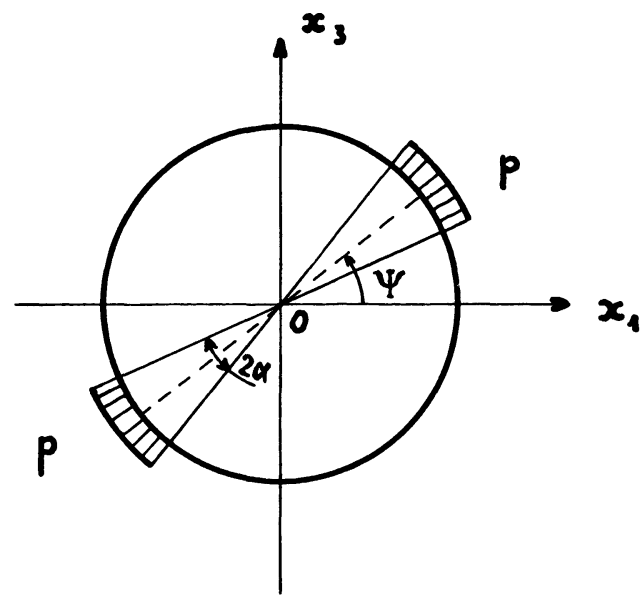

Fig. 2. - Cristal soumis à un secteur angulaire de compression.

[Cristal strained by edge angular sector stresses.]
Il a déjà été montré [5], pour la coupe $\mathrm{AT}$, que la variation relative de fréquence relative à ce type de contrainte s'écrit :

$$
\begin{aligned}
\frac{\bar{f}_{k}-f_{k}}{f_{k}}= & \frac{F}{2 c_{66} h_{0} \pi D} \times \\
& \times\left\{2\left(k_{3}-k_{1}\right) \frac{\sin 2 \alpha}{2 \alpha} \cos 2 \Psi-\left(k_{1}+k_{3}\right)\right\}
\end{aligned}
$$

où la symbolisation avec barre est relative à l'état final, $h_{0}$ est la demi-épaisseur de la plaque vibrante, $c_{66}$ le coefficient élastique du second ordre relatif à la vibration, et les $k_{i}$ des coefficients sans dimension fonctions des coefficients élastiques et de souplesse du second et du troisième ordre relatifs à la coupe AT.

Un coefficient de sensibilité à la compression a été introduit [5] sous la forme

$$
Q_{F}=2\left(k_{3}-k_{1}\right) \frac{\sin 2 \alpha}{2 \alpha} \cos 2 \Psi-\left(k_{1}+k_{3}\right) .
$$

Une généralisation de cette formule, correspondant aux contraintes étudiées, peut être proposée sous la forme

$$
\begin{aligned}
& Q_{F}(\theta, \varphi, \Psi, \alpha)= \\
& \quad=K_{1}(\theta, \varphi) \frac{\sin 2 \alpha}{2 \alpha} \cos (2 \Psi+\omega)+K_{2}(\theta, \varphi)
\end{aligned}
$$

où $K_{1}(\theta, \varphi)$ et $K_{2}(\theta, \varphi)$ sont des coefficients sans dimension, fonctions des coefficients élastiques et de souplesse du second et du troisième ordre de la coupe considérée. L'angle $\omega$ présente l'avantage de permettre le choix d'une origine quelconque pour l'angle $\Psi$.

Dans le cas d'un couple de forces appliquées ponctuellement, le coefficient de sensibilité $Q_{F}$ est relié au coefficient $K_{f}$ introduit par J. M. Ratajski, par la relation :

$$
Q_{F(\alpha \rightarrow 0)}=\frac{2 \pi f}{n} h_{0} c_{66} K_{f} .
$$

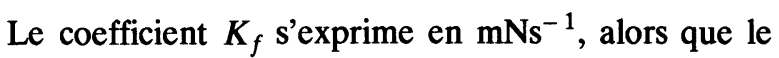
coefficient $Q_{F}$ présente l'avantage d'être sans dimension et d'introduire explicitement la valeur du secteur angulaire $2 \alpha$ ainsi que l'azimuth $\Psi$ d'application des forces.

2.2 Application a la coupe SC. - Contrairement à la coupe $\mathrm{AT}$, la coupe $\mathrm{SC}$ à double rotation admet, de par ses caractéristiques mécaniques («Stress Compensated»), un coefficient $K_{2}(\theta, \varphi)$ nul. Le coefficient de sensibilité à la compression $Q_{F}$ prend la forme simplifiée suivante :

$$
Q_{F}=K_{1}(\theta, \varphi) \frac{\sin 2 \alpha}{2 \alpha} \cos (2 \Psi+\omega)
$$

où $K_{1}(\theta, \varphi)$ dépend des coefficients élastiques et de souplesse du second et du troisième ordre de la coupe SC.

Pour que la fréquence du résonateur reste stable 
lorsque le cristal subit des variations de compression diamétrale, il suffit d'obtenir les conditions d'annulation de $Q_{F}$. Deux cas sont à envisager :

$1^{\text {er }}$ cas : $\cos (2 \Psi+\omega)=0$.

Il existe deux solutions :

ou

$$
\Psi_{1}=\frac{\pi}{4}-\frac{\omega}{2}
$$

$$
\Psi_{2}=\Psi_{1}+\pi
$$

Avec $\omega=\frac{\pi}{2}$ et $\alpha \rightarrow 0$, on retrouve les lieux des points de fixation proposés par A. Ballato [3] pour la coupe SC dans le cas particulier d'application des deux forces de compression diamétralement opposées (Fig. 3), ce qui confirme un résultat déjà connu.

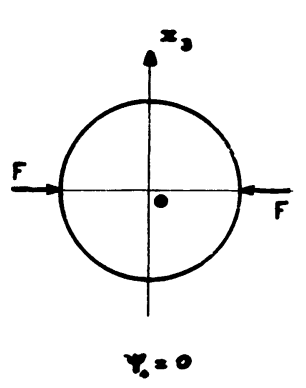

coupe SC

Fig. 3. - Positions optimales pour un couple de forces (coupe SC).

[Optimum positions for two strengths (SC-cut).]

$$
2^{\mathrm{e}} \operatorname{cas}: \frac{\sin 2 \alpha}{2 \alpha}=0
$$

Il existe une solution :

$$
2 \alpha=\pi \text {. }
$$

Lorsque la compression diamétrale du cristal est uniformément répartie sur toute sa périphérie (Fig. 4), le coefficient de sensibilité $Q_{F}$ reste nul quel que soit l'angle de positionnement.

Ce type de maintien appliqué à la coupe SC permet de rendre le résonateur insensible à toute variation de compression du cristal.

Des variations de température, de pression ou d'accélération peuvent être à l'origine de ces actions de compression.

\section{Sensibilité à la flexion.}

3.1 COEFFICIENT DE SENSIBILITE. - Lorsque le résonateur à quartz est soumis à une accélération axiale et que les conditions de l'indéformabilité relative du cristal par rapport à ses supports ne sont plus applicables, nous proposons d'interpréter sa sensibilité résiduelle par l'influence de sa déformation seule. Les

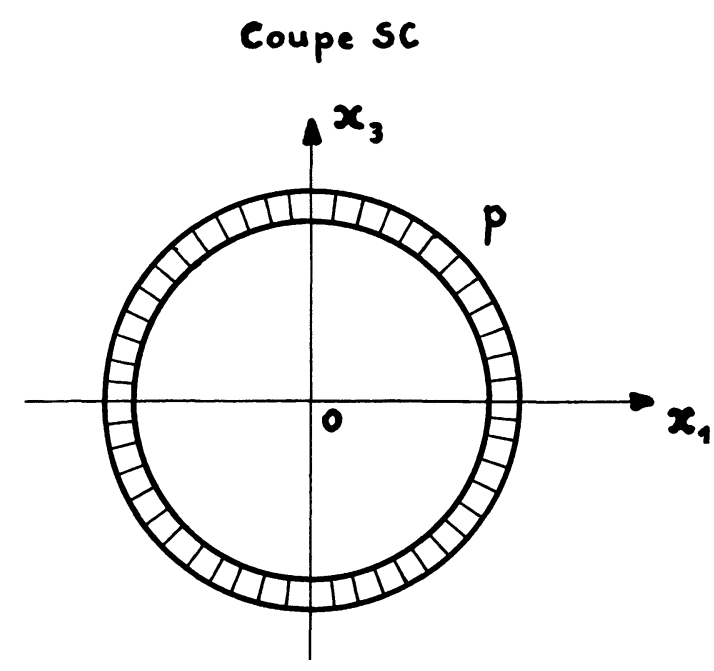

Fig. 4. - Cristal soumis à une compression uniforme.

[Cristal strained by edge stresses.]

autres effets, et notamment ceux des supports, ont été traités par ailleurs [8-10].

Le cristal étant relié à son environnement par une liaison non élastique, il subit alors une flexion en son centre suivant une surface sphérique ou une surface cylindrique. L'étude du coefficient de sensibilité à l'accélération a déjà été réalisée pour la coupe AT à simple rotation [11].

L'état de contrainte dans le quartz est déterminé pour une plaque mince circulaire et isotrope, avec une flèche maximale en son centre et une déformée symétrique par rapport à l'axe $\mathrm{x}_{2}$. Les contraintes de flexion étant nulles au niveau de la fibre moyenne, on fait l'hypothèse d'une variation de fréquence proportionnelle aux valeurs des contraintes surfaciques au centre du cristal.

Il est possible d'introduire un coefficient de sensibilité à l'accélération axiale $Q_{\mathrm{a}}$ sans dimension et dont la formulation générale est [11] :

$$
Q_{\mathrm{a}}=t_{i} \cdot f\left(K_{i}, \Psi\right)
$$

où $t_{i}$ est un coefficient non nul sans dimension, dépendant du type de liaison et $f\left(K_{i}, \Psi\right)$ une fonction des coefficients élastiques et de souplesse du second et du troisième ordre du quartz, et de la position $\Psi$ des liaisons relativement au repère cristallographique.

\subsection{APPlication a la COUPE SC.}

3.2.1 Flexion suivant une surface sphérique. - Des exemples de liaisons en appuis simples ou encastrés ont déjà été indiqués [11] pour obtenir cette condition. Les contraintes normales surfaciques $T_{1}$ et $T_{3}$ au centre de la plaque sont égales et indépendantes de la position des liaisons. Le coefficient de sensibilité à l'accélération axiale $Q_{\mathrm{a}}$ prend la forme simplifiée suivante :

$$
Q_{\mathrm{a}}=\left(k_{1}+k_{3}\right) t_{i}
$$


avec $k_{1}$ et $k_{3}$ deux constantes non nulles fonctions des coefficients élastiques et de souplesse du second et du troisième ordre de la coupe SC.

La valeur de $t_{i}$ dépendant du type de liaison, la solution réalisant l'encastrement sur toute la périphérie du cristal (Fig. 5) permettent d'obtenir la plus faible valeur de $t_{i}$, donc de $Q_{\mathrm{a}}$.

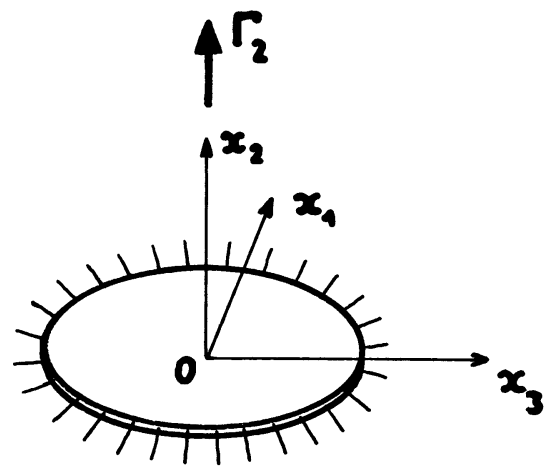

Fig. 5. - Cristal encastré soumis à une accélération axiale.

[Axial acceleration applied to an embeded crystal.]

3.2.2 Flexion suivant une surface cylindrique. - C'est le cas du cristal en appui simple ou encastré en deux points diamétralement opposés. La position des liaisons, par rapport au repère cristallographique, intervient dans le calcul de sensibilité et les contraintes surfaciques $T_{1}$ et $T_{3}$ ne sont pas égales.

En tenant compte de la position des liaisons, les contraintes au centre du cristal sont données en fonction de l'accélération axiale $\Gamma_{2}$ par :

$$
\begin{aligned}
& T_{1}=2 \frac{\rho R^{2}}{h_{0}}(1+\cos 2 \Psi) \Gamma_{2} \\
& T_{3}=2 \frac{\rho R^{2}}{h_{0}}(1-\cos 2 \Psi) \Gamma_{2} \\
& T_{5}=2 \frac{\rho R^{2}}{h_{0}} \frac{1}{2}(\sin 2 \Psi) \Gamma_{2}
\end{aligned}
$$

où $\rho$ est la masse volumique du quartz.

Le coefficient de sensibilité à l'accélération axiale $Q_{\mathrm{a}}$ devient

$$
Q_{\mathrm{a}}=4\left\{\left(k_{1}-k_{3}\right) \cos 2 \Psi+\frac{k_{5}}{2} \sin 2 \Psi+\left(k_{1}+k_{3}\right)\right\}
$$

avec $k_{5}$ une constante non nulle fonction des coefficients élastiques et de souplesse du second et du troisième ordre de la coupe SC.

En remplaçant les valeurs de $\cos 2 \Psi$ et $\sin 2 \Psi$ en fonction de $\tan \Psi$ et en posant $t=\tan \Psi, Q_{\mathrm{a}}$ s'écrit :

$$
Q_{\mathrm{a}}=\frac{4}{\left(1+t^{2}\right)}\left(2 k_{3} t^{2}+k_{5} t+2 k_{1}\right)
$$

avec les coefficients $k_{1}, k_{3}$ et $k_{5}$ tels que :

$$
\begin{gathered}
k_{1}=\left(c_{661}+2 c_{66}\right) S_{11}+c_{662} S_{21}+c_{663} S_{31}+ \\
+c_{664} S_{41}+c_{665} S_{51}+c_{666} S_{61} \\
k_{3}=\left(c_{661}+2 c_{66}\right) S_{13}+c_{662} S_{23}+c_{663} S_{33}+ \\
+c_{664} S_{43}+c_{665} S_{53}+c_{666} S_{63} \\
k_{5}=\left(c_{661}+2 c_{66}\right) S_{15}+c_{662} S_{25}+c_{663} S_{35}+ \\
+ \\
+c_{664} S_{45}+c_{665} S_{55}+c_{666} S_{65}
\end{gathered}
$$

où $c_{i j}$ et $s_{i j}$ sont respectivement les coefficients élastiques et de souplesse du second ordre et $c_{i j k}$ les coefficients élastiques du troisième ordre de la coupe SC.

Les valeurs numériques sont calculées pour une température de $25^{\circ} \mathrm{C}$

$$
\begin{aligned}
& k_{1}=0,576 \\
& k_{3}=0,098 \\
& k_{5}=-1,094 .
\end{aligned}
$$

Pour que la fréquence reste stable lorsque le résonateur subit une accélération axiale, il faut obtenir l'annulation de $Q_{\mathrm{a}}$, soit

$$
2 k_{3} t^{2}+k_{5} t+2 k_{1}=0 .
$$

Cette équation du second degré en $t$ admet deux racines réelles

$$
t=\frac{1}{4 k_{3}}\left\{-k_{5} \pm\left(k_{5}^{2}-16 k_{1} k_{3}\right)^{1 / 2}\right\}
$$

qui prennent les valeurs suivantes

$$
\begin{aligned}
& t_{1}=4,173 \\
& t_{2}=1,408 .
\end{aligned}
$$

Les positions, permettant l'annulation de la sensibilité accélérométrique axiale pour maintien en deux points opposés, sont obtenues à $25^{\circ} \mathrm{C}$

$$
\Psi_{1}=76,52^{\circ}
$$

ou

$$
\Psi_{2}=54,62^{\circ} \text {. }
$$

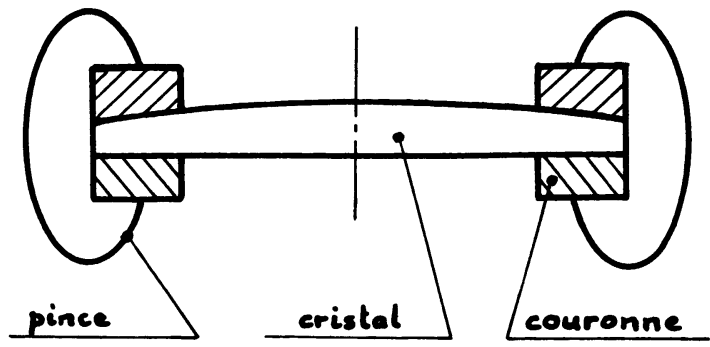

Fig. 6. - Schéma simplifié de la structure du résonateur. [Simplified scheme of resonator structure.] 


\section{Conclusion.}

Comme dans le cas de la coupe AT, il n'existe pas pour la coupe SC de solution permettant d'annuler simultanément les effets de la compression et de la flexion, lorsqu'une fixation classique par deux points diamétralement opposés est mise en œuvre.

Le meilleur compromis est celui qui permet d'annuler les effets de la compression en réduisant au minimum ceux de la flexion sans toutefois annuler complètement ces derniers. Ceci peut être obtenu par une structure de maintien réalisant l'encastrement périphérique intégral $\mathrm{du}$ cristal résonnant de coupe SC. Pour obtenir ce résultat une solution consiste à maintenir le cristal entre deux couronnes de quartz de coupe SC [12], les deux couronnes étant plaquées contre le cristal à l'aide d'un jeu de pinces élastiques (Fig. 6). Les variations de serrages sont sans effet sur la fréquence puisque répercutées sur toute la périphérie du cristal résonnant.

\section{Bibliographie}

[1] VAlentin, J. P., Influence des phénomènes accélérométriques sur la fréquence des résonateurs à quartz embarqués. Bul. BNM, $\mathrm{n}^{\text {os }} 63-64,2$ (1986).

[2] Perdigues, J. P., Etude de l'influence des forces sur la fréquence d'un résonateur à quartz. Thèse de Docteur-Ingénieur, Faculté des Sciences de Besançon, 1975.

[3] Ballato, A. and Mizan, M., Simplified expressions for the stress-frequency coefficients of quartz plates. IEEE Trans. SU 31, n 1 (1984).

[4] Besson, R. J., Résonateur piézoélectrique à cristal autosuspendu. Brevet d'invention $\mathrm{n}^{\circ} 7802261$, 1978.

[5] Delaite, R. and Valentin, J. P., Analysis of forcefrequency effect of piezoelectric circular resonators strained by edge angular-sector stresses. Il Nuovo Cimento 6D, $\mathrm{n}^{\circ} 6$ (1985).

[6] Eernisse, E. P., Quartz resonator frequency shifts arising from electrode stress. 29th Annual Frequency Control Symposium, Fort Monmouth, 1975.
[7] RAtAJSKI, J. M., Force-frequency coefficient of singly rotated vibrating quartz cristals. IBM J. Res. Dev. 12 (1968).

[8] JANIAUD, D., Modélisation de l'influence d'une accélération sur la fréquence des résonateurs à quartz. Thèse de Docteur-Ingénieur, Faculté des Sciences de Besançon, 1978.

[9] Besson, R., Gagnepain, J. J., Janiaud, D. and VALDOIS, M., Design of a bulk wave quartz resonator insensitive to acceleration. Proc. 33rd Annual Frequency Control Symposium, Fort Monmouth May-June, 1979.

[10] Delaite, R., Influence de la structure de maintien sur la sensibilité accélérométrique et barométrique des résonateurs à quartz de haute stabilité. Thèse de Doctorat, $\mathrm{N}^{\circ} 005$, Université de Franche-Comté, Besançon, 1985.

[11] DElaite, R., Résonateur à quartz pour environnement sévère, Revue Phys. Appl. 20 (1985).

[12] Delaite, R., Résonateur piézoélectrique à extrémum de sensibilité vis-à-vis des contraintes extérieures de pression. Brevet d'invention Français, $\mathrm{N}^{\circ} 85 / 09058$ déposé le 14 juin 1985. 